\title{
Shortcuts to adiabaticity for non-Hermitian systems
}

\author{
S. Ibáñez, ${ }^{1}$ S. Martínez-Garaot, ${ }^{1}$ Xi Chen, ${ }^{1,2}$ E. Torrontegui, ${ }^{1}$ and J. G. Muga ${ }^{1}$ \\ ${ }^{1}$ Departamento de Química Física, Universidad del País Vasco-Euskal Herriko Unibertsitatea, Apdo. 644, Bilbao, Spain \\ ${ }^{2}$ Department of Physics, Shanghai University, 200444 Shanghai, China
}

(Received 14 June 2011; revised manuscript received 3 August 2011; published 29 August 2011)

\begin{abstract}
Adiabatic processes driven by non-Hermitian, time-dependent Hamiltonians may be sped up by generalizing inverse engineering techniques based on counter-diabatic (transitionless driving) algorithms or on dynamical invariants. We work out the basic theory and examples described by two-level Hamiltonians: the acceleration of rapid adiabatic passage with a decaying excited level and of the dynamics of a classical particle on an expanding harmonic oscillator.
\end{abstract}

DOI: 10.1103/PhysRevA.84.023415

PACS number(s): 32.80.Qk, 42.50.-p

\section{INTRODUCTION}

There is nowadays considerable interest in speeding up adiabatic processes. Adiabatic methods to manipulate and prepare states, in principle in a robust way, are ubiquitous in atomic and molecular physics, nuclear magnetic resonance, optics, and other fields, but the times required may be too long for some applications. Moreover, the ideal robustness may be spoiled by the accumulation of perturbations and decoherence due to noise and undesired interactions. We refer to fast time-dependent processes that reproduce the effect of a slow, adiabatic driving of a quantum system as "shortcuts to adiabaticity" [1-27]. We also apply the term to the inverse engineering methods used to design these processes. In the adiabatic process of reference, the external control parameters are modified slowly from some initial configuration to a final one. In the corresponding shortcut, the system is driven in a predetermined short time to a final state that reproduces in the instantaneous basis the initial populations, as the adiabatic process would do, but possibly allowing for some transient excitation along the way. Studies and experiments to speed up adiabatic processes have been carried out for transport [4-6,12,26,27], wave splitting [8,9], trap expansions and compressions [7,11,13,15-21,23-25], or internal state control [1-3,10,14,22]. These studies have so far been performed for Hermitian Hamiltonians, but many systems admit an effective non-Hermitian description. In this paper, we put forward shortcuts to adiabaticity techniques for non-Hermitian Hamiltonians. Specifically, we shall generalize the inverse engineering method proposed by Demirplak, Rice [1-3], and Berry [10], and the one based on dynamical invariants [7,13]. While these methods are intimately connected, as shown in Ref. [22], and in fact potentially equivalent, in standard applications they are used in different ways and provide different answers, so we shall consider them separately here. As study cases, we shall discuss the laser-driven, fast population inversion of a two-level decaying atom and the motion of a classical particle in a harmonic oscillator with time-dependent frequency.

\section{A. Non-Hermitian Hamiltonians: Basic formulas}

Non-Hermitian Hamiltonians typically describe subsystems of a larger system [28]. We shall first review a basic set of relations and notation [28] for a non-Hermitian time-dependent Hamiltonian $H_{0}(t)$ with $N$ nondegenerate right eigenstates $\{|n(t)\rangle\}, n=1,2 \ldots, N$,

$$
H_{0}(t)|n(t)\rangle=E_{n}(t)|n(t)\rangle
$$

and biorthogonal partners $\{|\hat{n}(t)\rangle\}$,

$$
H_{0}^{\dagger}(t)|\hat{n}(t)\rangle=E_{n}^{*}(t)|\hat{n}(t)\rangle
$$

where the asterisk means "complex conjugate" and the dagger denotes the adjoint operator. They satisfy

$$
\langle\hat{n}(t) \mid m(t)\rangle=\delta_{n m}
$$

and the closure relations

$$
\sum_{n}|\hat{n}(t)\rangle\left\langle n(t)\left|=\sum_{n}\right| n(t)\right\rangle\langle\hat{n}(t)|=1 .
$$

$\langle\hat{n}(t)|$ is the left eigenvector of $H_{0}(t)$,

$$
\langle\hat{n}(t)| H_{0}(t)=\langle\hat{n}(t)| E_{n}(t),
$$

and $\langle n(t)|$ the left eigenvector of $H_{0}^{\dagger}(t)$,

$$
\langle n(t)| H_{0}^{\dagger}(t)=\langle n(t)| E_{n}^{*}(t) .
$$

We can thus write the Hamiltonian and its adjoint as

$$
\begin{aligned}
& H_{0}(t)=\sum_{n}|n(t)\rangle E_{n}(t)\langle\hat{n}(t)|, \\
& H_{0}^{\dagger}(t)=\sum_{n}|\hat{n}(t)\rangle E_{n}^{*}(t)\langle n(t)| .
\end{aligned}
$$

The time-dependent Schrödinger equations for a generic state $|\Psi(t)\rangle$ and for its biorthogonal partner $|\hat{\Psi}(t)\rangle$ satisfying $\langle\hat{\Psi}(t) \mid \Psi(t)\rangle=1$ are

$$
\begin{aligned}
i \hbar \partial_{t}|\Psi(t)\rangle & =H_{0}(t)|\Psi(t)\rangle, \\
i \hbar \partial_{t}|\hat{\Psi}(t)\rangle & =H_{0}^{\dagger}(t)|\hat{\Psi}(t)\rangle .
\end{aligned}
$$

\section{ADIABATICITY FOR NON-HERMITIAN HAMILTONIANS}

Before studying the shortcuts, we need to set the adiabatic approximation when $H_{0}(t)$ is non-Hermitian [29,30]. A general time-dependent state $|\Psi(t)\rangle$ is a linear combination of instantaneous (right) eigenvectors $|n(t)\rangle$ of $H_{0}(t)$ with time-dependent coefficients. Similarly, $|\hat{\Psi}(t)\rangle$ is a linear combination of instantaneous (right) eigenvectors $|\hat{n}(t)\rangle$ of 
$H_{0}^{\dagger}(t)$. In the adiabatic approximation, if only one of these eigenvectors is populated initially, it will remain so throughout the time evolution. In other words, we consider approximate solutions of Eqs. (8) and (9) of the form

$$
\begin{aligned}
\left|\varphi_{n}(t)\right\rangle & =e^{i \beta_{n}(t)}|n(t)\rangle, \\
\left|\hat{\varphi}_{n}(t)\right\rangle & =e^{i \hat{\beta}_{n}(t)}|\hat{n}(t)\rangle .
\end{aligned}
$$

Introducing these two vectors into Eqs. (8) and (9), respectively, we have

$$
\begin{aligned}
& i \dot{\beta}_{n}|n(t)\rangle+\left|\partial_{t} n(t)\right\rangle=\frac{H_{0}(t)}{i \hbar}|n(t)\rangle, \\
& i \dot{\hat{\beta}}_{n}|\hat{n}(t)\rangle+\left|\partial_{t} \hat{n}(t)\right\rangle=\frac{H_{0}^{\dagger}(t)}{i \hbar}|\hat{n}(t)\rangle,
\end{aligned}
$$

where the dot denotes the derivative with respect to time. In general, Eqs. (12) and (13) are only approximately correct since the solution Ansätze, Eqs. (10) and (11), are only approximate. Multiplying Eq. (12) by $\langle\hat{n}(t)|$ and Eq. (13) by $\langle n(t)|$, taking into account Eqs. (1) and (2), and integrating, we find

$$
\begin{aligned}
& \beta_{n}(t)=\int_{0}^{t}\left[\frac{-E_{n}\left(t^{\prime}\right)}{\hbar}+i\left\langle\hat{n}\left(t^{\prime}\right) \mid \partial_{t^{\prime}} n\left(t^{\prime}\right)\right\rangle\right] d t^{\prime}, \\
& \hat{\beta}_{n}(t)=\int_{0}^{t}\left[\frac{-E_{n}^{*}\left(t^{\prime}\right)}{\hbar}+i\left\langle n\left(t^{\prime}\right) \mid \partial_{t^{\prime}} \hat{n}\left(t^{\prime}\right)\right\rangle\right] d t^{\prime},
\end{aligned}
$$

where the initial phases are set to zero. As $\left\langle n(t) \mid \partial_{t} \hat{n}\right\rangle^{*}=$ $\left\langle\partial_{t} \hat{n} \mid n(t)\right\rangle$ and, from Eq. (3), $\left\langle\partial_{t} \hat{n} \mid n(t)\right\rangle=-\left\langle\hat{n}(t) \mid \partial_{t} n\right\rangle$, we have that $\hat{\beta}_{n}=\beta_{n}^{*}$. $\beta_{n}(t)$ and $\hat{\beta}_{n}(t)$ are the, generally complex, adiabatic phases (with a dynamical part depending on the instantaneous eigenvalue and a geometric part) that generalize the real adiabatic phase of Hermitian systems [31].

\section{A. Adiabaticity condition for time-dependent non-Hermitian} Hamiltonians

The adiabaticity condition for time-dependent Hermitian Hamiltonians is given by

$$
\left|\left\langle n(t) \mid \partial_{t} m(t)\right\rangle\right| \ll \frac{1}{\hbar}\left|E_{n}(t)-E_{m}(t)\right|, n \neq m,
$$

in terms of instantaneous eigenstates and eigenvalues. Following closely its derivation in Ref. [32], we may generalize it for non-Hermitian Hamiltonians as

$$
\left|\left\langle\hat{n}(t) \mid \partial_{t} m(t)\right\rangle\right| \ll \frac{1}{\hbar}\left|E_{n}(t)-E_{m}(t)\right|, n \neq m .
$$

To that end we start with the expansion

$$
|\Psi(t)\rangle=\sum_{n} a_{n}(t) e^{\frac{1}{i \hbar} \int_{0}^{t} E_{n}\left(t^{\prime}\right) d t^{\prime}}|n(t)\rangle,
$$

which, inserted into Eq. (8) gives a system of differential equations for the amplitudes,

$$
\begin{aligned}
\dot{a}_{n}(t)= & \sum_{k \neq n} \frac{a_{k}(t)}{\hbar \omega_{n k}(t)}\left[\exp \left(i \int_{0}^{t} \omega_{n k}\left(t^{\prime}\right) d t^{\prime}\right)\right] \\
& \times\left\langle\hat{n}(t)\left|\frac{\partial H}{\partial t}\right| k(t)\right\rangle,
\end{aligned}
$$

where $\omega_{n k}(t):=\left[E_{n}(t)-E_{k}(t)\right] / \hbar$. Then we take $|\Psi(0)\rangle=$ $|m(0)\rangle$ at $t=0$, make the approximation $a_{k}(t)=\delta_{k m}$ on the right-hand side of Eq. 19, use $\left\langle\hat{n}(t)\left|\frac{\partial H}{\partial t}\right| m(t)\right\rangle=\left[E_{m}(t)-\right.$ $\left.E_{n}(t)\right]\left\langle\hat{n}(t) \mid \partial_{t} m(t)\right\rangle$, and integrate to get

$$
\left|a_{n}\right| \sim\left|\frac{\hbar\left\langle\hat{n}(t) \mid \partial_{t} m(t)\right\rangle}{\left[E_{n}(t)-E_{m}(t)\right]}\right|, n \neq m .
$$

The adiabaticity condition requires that this is much smaller than 1, namely, Eq. (17).

\section{TRANSITIONLESS DRIVING ALGORITHM}

In Refs. [1-3], Demirplak and Rice, and later Berry [10], proposed a method to design a Hermitian Hamiltonian $H(t)$ for which the approximate adiabatic dynamics driven by the Hermitian Hamiltonian $H_{0}(t)$ becomes exact. We shall generalize this method for non-Hermitian Hamiltonians.

As in Ref. [10], we now impose that all $\left|\varphi_{n}(t)\right\rangle$ given by Eqs. (10) and (14) satisfy exactly the Schrödinger equation for a yet unknown $H(t)$,

$$
i \hbar \partial_{t}\left|\varphi_{n}(t)\right\rangle=H(t)\left|\varphi_{n}(t)\right\rangle
$$

Similarly,

$$
i \hbar \partial_{t}\left|\hat{\varphi}_{n}(t)\right\rangle=H^{\dagger}(t)\left|\hat{\varphi}_{n}(t)\right\rangle .
$$

The states $\left|\varphi_{n}(t)\right\rangle$ and $\left|\hat{\varphi}_{n}(t)\right\rangle$ can be written in terms of the corresponding evolution operators $U(t)$ and $\hat{U}(t)$,

$$
\begin{aligned}
\left|\varphi_{n}(t)\right\rangle & =U(t)|n(0)\rangle, \\
\left|\hat{\varphi}_{n}(t)\right\rangle & =\hat{U}(t)|\hat{n}(0)\rangle .
\end{aligned}
$$

The Hamiltonian $H(t)$ can be found from

$$
i \hbar \partial_{t} U(t)=H(t) U(t),
$$

as

$$
H(t)=i \hbar \partial_{t} U(t) \hat{U}^{\dagger}(t)
$$

since $\hat{U}^{\dagger}(t) U(t)=1_{\text {op }}$ [28]. The evolution operators can be written as

$$
\begin{aligned}
& U(t)=\sum_{n} e^{i \beta_{n}(t)}|n(t)\rangle\langle\hat{n}(0)|, \\
& \hat{U}(t)=\sum_{n} e^{i \hat{\beta}_{n}(t)}|\hat{n}(t)\rangle\langle n(0)| .
\end{aligned}
$$

Using now Eq. (25),

$$
H(t)=H_{0}(t)+H_{1}(t),
$$

where

$$
\begin{aligned}
H_{1}(t)= & i \hbar \sum_{n}\left[\left|\partial_{t} n(t)\right\rangle\langle\hat{n}(t)|\right. \\
& \left.-\left\langle\hat{n}(t) \mid \partial_{t} n(t)\right\rangle|n(t)\rangle\langle\hat{n}(t)|\right] .
\end{aligned}
$$

$H(t)$ drives the system along the adiabatic paths defined by $H_{0}(t)$.

As noted in Refs. [10] and [22], this Hamiltonian is not unique. For a given set $\{|n(t)\rangle\}$, the same final populations are found by choosing different phases. Let us rewrite $\left|\varphi_{n}(t)\right\rangle$ and $\left|\hat{\varphi}_{n}(t)\right\rangle$ in terms of arbitrary phases, $\xi_{n}(t)$ and $\hat{\xi}_{n}(t)$, which we now regard as manipulable functions obeying $\xi_{n}(t)=\hat{\xi}_{n}^{*}(t)$ so that $\left\langle\hat{\varphi}_{n}(t) \mid \varphi_{n}(t)\right\rangle=1$,

$$
\left|\varphi_{n}(t)\right\rangle=e^{i \xi_{n}(t)}|n(t)\rangle,\left|\hat{\varphi}_{n}(t)\right\rangle=e^{i \hat{\xi}_{n}(t)}|\hat{n}(t)\rangle .
$$


We assume $\xi_{n}(0)=\hat{\xi}_{n}(0)=0$ and define the new evolution operators

$$
\begin{aligned}
& U_{\xi}(t)=\sum_{n} e^{i \xi_{n}(t)}|n(t)\rangle\langle\hat{n}(0)|, \\
& \hat{U}_{\xi}(t)=\sum_{n} e^{i \hat{\xi}_{n}(t)}|\hat{n}(t)\rangle\langle n(0)| .
\end{aligned}
$$

From Eq. (25), the corresponding Hamiltonian becomes

$$
H_{\xi}(t)=-\hbar \sum_{n}|n(t)\rangle \dot{\xi}_{n}(t)\left\langle\hat{n}(t)\left|+i \hbar \sum_{n}\right| \partial_{t} n(t)\right\rangle\langle\hat{n}(t)| .
$$

\section{TRANSITIONLESS DRIVING ALGORITHM APPLIED TO A DECAYING TWO-LEVEL ATOM}

\section{A. $H_{1}(t)$ applied to a decaying two-level atom}

As an application of the general approach of the previous section, we shall speed up adiabatic processes in a two-level atom with spontaneous decay illuminated by a chirped laser pulse, i.e., one with a time-dependent frequency. A particular case of practical importance is population inversion, which generalizes the shortcut techniques described for a Hermitian two-level system [1-3,10,14].

If the decayed atom escapes from the trap by recoil, a Hamiltonian (rather than master equation) description is enough for the trapped atom [33,34]. We shall also assume a semiclassical treatment of the interaction between a laser electric field linearly polarized and a decay rate (inverse life-time) $\Gamma$ from the excited state.

Applying the electric dipole approximation, a laser-adapted interaction picture, and the rotating wave approximation, the Hamiltonian, disregarding atomic motion, is (see, e.g., Ref. [35]),

$$
H_{a 0}(t)=\frac{\hbar}{2}\left(\begin{array}{cc}
-\Delta(t) & \Omega_{R}(t) \\
\Omega_{R}(t) & \Delta(t)-i \Gamma
\end{array}\right)
$$

in the atomic basis $|1\rangle=\left(\begin{array}{l}1 \\ 0\end{array}\right),|2\rangle=\left(\begin{array}{l}0 \\ 1\end{array}\right)$. In this description, the norm of the state decreases since, if an atom decays spontaneously from level 2 , it is eliminated from the quantum ensemble. The detuning from the atomic transition frequency $\omega_{0}$ is $\Delta(t)=\omega_{0}-\omega_{i}(t)$, where $\omega_{i}(t)$ is the time-dependent instantaneous field frequency. We consider a pulse with slowly varying envelope so that the (real) Rabi frequency $\Omega_{R}(t)$ depends on time too. In the example below, we shall take $\Gamma$ as a constant, although, in a general case, it could also depend on time, $\Gamma=\Gamma(t)$, as an effective decay rate controlled by further interactions; see, e.g., Ref. [34]. The eigenvalues of this Hamiltonian are

$$
E_{ \pm}(t)=\frac{\hbar}{4}\left\{-i \Gamma \pm \sqrt{-[\Gamma+2 i \Delta(t)]^{2}+4 \Omega_{R}^{2}(t)}\right\}
$$

and the normalized eigenstates are

$$
\begin{aligned}
& \left|\chi_{+}(t)\right\rangle=\sin \left(\frac{\alpha}{2}\right)|1\rangle+\cos \left(\frac{\alpha}{2}\right)|2\rangle, \\
& \left|\chi_{-}(t)\right\rangle=\cos \left(\frac{\alpha}{2}\right)|1\rangle-\sin \left(\frac{\alpha}{2}\right)|2\rangle,
\end{aligned}
$$

where the mixing angle $\alpha=\alpha(t)$ is complex and defined as

$$
\tan \alpha=\frac{\Omega_{R}}{\Delta-i \Gamma}
$$

The adjoint of $H_{a 0}(t)$ is

$$
H_{a 0}^{\dagger}(t)=\frac{\hbar}{2}\left(\begin{array}{cc}
-\Delta(t) & \Omega_{R}(t) \\
\Omega_{R}(t) & \Delta(t)+i \Gamma
\end{array}\right),
$$

with eigenvalues $E_{ \pm}^{*}(t)$ and normalized eigenstates

$$
\begin{aligned}
\left|\hat{\chi}_{+}(t)\right\rangle & =\sin \left(\frac{\alpha^{*}}{2}\right)|1\rangle+\cos \left(\frac{\alpha^{*}}{2}\right)|2\rangle, \\
\left|\hat{\chi}_{-}(t)\right\rangle & =\cos \left(\frac{\alpha^{*}}{2}\right)|1\rangle-\sin \left(\frac{\alpha^{*}}{2}\right)|2\rangle .
\end{aligned}
$$

Note that the coefficients are complex conjugate of those in Eq. (34) because $H_{a 0}(t)$ is equal to its transpose [28].

For this system, the supplementary Hamiltonian in Eq. (28) takes the form

$$
\begin{aligned}
H_{a 1}(t)= & i \hbar\left[\left|\partial_{t} \chi_{+}(t)\right\rangle\left\langle\hat{\chi}_{+}(t)\right|\right. \\
& -\left\langle\hat{\chi}_{+}(t) \mid \partial_{t} \chi_{+}(t)\right\rangle\left|\chi_{+}(t)\right\rangle\left\langle\hat{\chi}_{+}(t)\right| \\
& +\left|\partial_{t} \chi_{-}(t)\right\rangle\left\langle\hat{\chi}_{-}(t)\right| \\
& \left.-\left\langle\hat{\chi}_{-}(t) \mid \partial_{t} \chi_{-}(t)\right\rangle\left|\chi_{-}(t)\right\rangle\left\langle\hat{\chi}_{-}(t)\right|\right],
\end{aligned}
$$

where, according to Eqs. (34) and (37),

$$
\begin{aligned}
& \left\langle\hat{\chi}_{ \pm}(t) \mid \partial_{t} \chi_{ \pm}(t)\right\rangle=0, \\
& \left\langle\hat{\chi}_{\mp}(t) \mid \partial_{t} \chi_{ \pm}(t)\right\rangle= \pm \frac{\dot{\alpha}}{2},
\end{aligned}
$$

So

$$
H_{a 1}(t)=\hbar\left(\begin{array}{cc}
0 & C(t) \\
-C(t) & 0
\end{array}\right),
$$

where $C(t)=i \dot{\alpha} / 2$ and

$$
\dot{\alpha}=\frac{\dot{\Omega}_{R}[\Delta(t)-i \Gamma / 2]-\Omega_{R}(t)(\dot{\Delta}-i \dot{\Gamma} / 2)}{[\Delta(t)-i \Gamma / 2]^{2}+\Omega_{R}^{2}(t)} .
$$

Then, the Hamiltonian $H_{a}(t)=H_{a 0}+H_{a 1}$ takes the form

$$
H_{a}(t)=\frac{\hbar}{2}\left(\begin{array}{cc}
-\Delta(t) & \Omega_{R}(t)+2 C(t) \\
\Omega_{R}(t)-2 C(t) & \Delta(t)-i \Gamma
\end{array}\right) .
$$

The practical realization of this Hamiltonian is not straightforward. In particular, the off-diagonal terms are not the complex conjugate of each other unless the real part of $C(t)$ becomes zero, so in general there is no simple laser interaction leading to Eq. (42). We shall explore in the following subsection the possibility to avoid this result by playing with different phases, as in Eq. (31).

\section{B. $H_{\xi}(t)$ applied to a decaying two-level atom}

For the decaying two-level atom, using Eq. (31) with phases $\xi_{+}=\xi_{+}(t)$ and $\xi_{-}=\xi_{-}(t)$ associated with $\left|\chi_{+}(t)\right\rangle$ and $\left|\chi_{-}(t)\right\rangle$, we find

$$
\begin{aligned}
& H_{\xi a}(t) / \hbar= \\
& \qquad\left[\begin{array}{cc}
-\sin ^{2}\left(\frac{\alpha}{2}\right) \dot{\xi}_{+}-\cos ^{2}\left(\frac{\alpha}{2}\right) \dot{\xi}_{-} & \frac{\sin \alpha}{2}\left(\dot{\xi}_{-}-\dot{\xi}_{+}\right)+C \\
\frac{\sin \alpha}{2}\left(\dot{\xi}_{-}-\dot{\xi}_{+}\right)-C & -\cos ^{2}\left(\frac{\alpha}{2}\right) \dot{\xi}_{+}-\sin ^{2}\left(\frac{\alpha}{2}\right) \dot{\xi}_{-}
\end{array}\right] .
\end{aligned}
$$


The phases in the matrix elements $H_{\xi a, 12}(t)$ and $H_{\xi a, 21}(t)$ only affect the first terms, which are equal. Therefore, in general, the manipulation of the phases is not enough to make the nondiagonal terms complex conjugate of each other since this requires not only $\operatorname{Im}\left[\left(\dot{\xi}_{-}-\dot{\xi}_{+}\right) \sin \alpha\right]=0$ but $\operatorname{Re}[C(t)]=0$, too. We also add potentially complex terms in the diagonal that again could complicate the physical realization.

In summary, the phase manipulation does not help to implement the shortcut. In some parameter regimes, however, an approximation to $H_{a 1}$ that leads to essentially the same results may be easily realized, as discussed next.

\section{Forced population inversion}

We study now the laser-driven coherent decay from the upper level of a two-level system with slow spontaneous decay. This type of decay process is of interest as it occurs coherently, unlike the incoherent spontaneous emission. The coherent decay may be driven adiabatically with a "rapid" adiabatic passage (RAP) technique [36], sweeping the laser frequency across resonance. The adjective "rapid" here could be misleading: it simply means "faster than the spontaneous decay," but as the approach is based on an adiabatic passage, it fails for short enough pulse times.

For the two-level decaying atom, the adiabaticity condition, Eq. (17), is

$$
\left|\left\langle\hat{\chi}_{+}(t) \mid \partial_{t} \chi_{-}(t)\right\rangle\right| \ll \frac{1}{\hbar}\left|E_{+}(t)-E_{-}(t)\right| .
$$

Introducing here Eqs. (33), (34), and (37), it takes the form

$$
|\dot{\alpha}(t)| \ll|\Omega(t)|
$$

where $\Omega(t)=\sqrt{-[\Gamma+2 i \Delta(t)]^{2}+4 \Omega_{R}^{2}(t)}$. We shall provide an example in which this condition fails and then apply the Hamiltonian in Eq. (42) to remedy this problem and achieve a fast full decay.

We consider a linearly chirped Gaussian pulse with detuning $\Delta(t)=\omega_{0}-\omega_{i}(t)=-2 B t$ and Gaussian Rabi frequency $\Omega_{R}(t)=\Omega_{0} e^{-A t^{2}}$.

The initial conditions for the populations of the ground and excited states are $P_{1}(0)=0$ and $P_{2}(0)=1$. In Fig. 1, we show that the application of a RAP pulse with $H_{a 0}(t)$ is only partially successful. Note the slow spontaneous decay before and after

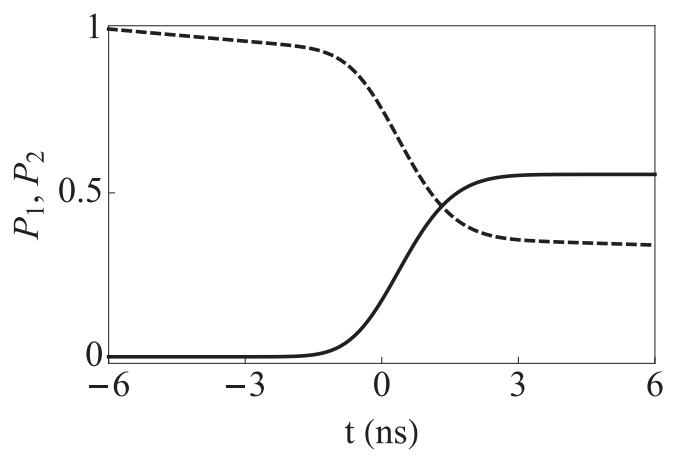

FIG. 1. Population of the ground state, $P_{1}(t)$ (solid line), and of the excited state, $P_{2}(t)$ (dashed line), for the Hamiltonian $H_{a 0}(t)$. Parameters: $\Gamma=2 \pi \times 2 \mathrm{MHz}, A=(2 \pi)^{2} \times 0.01 \mathrm{GHz}^{2}$, $B=(2 \pi)^{2} \times 0.00025 \mathrm{GHz}^{2}$, and $\Omega_{0}=2 \pi \times 100 \mathrm{MHz}$.

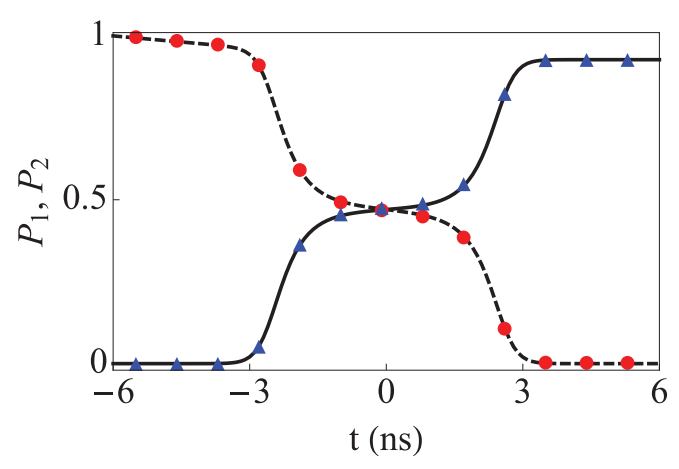

FIG. 2. (Color online) Population of the ground state, $P_{1}(t)$ (solid line), and of the excited state, $P_{2}(t)$ (dashed line), for the total Hamiltonian $H_{a}(t)$, coinciding with the populations $P_{1}(t)$ (triangles) and $P_{2}(t)$ (circles) when $H_{a}(t)$ is approximated by neglecting $\operatorname{Re}[C(t)]$. Parameters as described in the legend of Fig. 1.

the pulse and a faster forced transition during the pulse around $t=0$. The pulse duration is too short and adiabaticity fails. Figure 2 shows the fast full population inversion when adding the supplementary Hamiltonian $H_{a 1}(t)$, Eq. (40). This Hamiltonian has off-diagonal terms with real and imaginary parts depicted in Fig. 3. Whereas the imaginary parts, the bigger bumps in Fig. 3, are realizable by a complementary laser with orthogonal polarization [14], the real parts constitute a non-Hermitian contribution. They are, however, small, and an approximation of $H_{a 1}(t)$ neglecting them provides essentially the same dynamics, as shown in Fig. 2. This remains valid in the strong-driving regime in which $\Gamma \ll \Omega_{0}$ and the natural lifetime is large compared to the duration of the forced decay. Until a physical implementation of the real parts is discovered, the application of this shortcut technique for population inversion with spontaneous decay must therefore be limited to this regime.

\section{INVARIANTS-BASED INVERSE ENGINEERING}

Lewis and Riesenfeld [37] proposed the use of dynamical invariants of a quantum mechanical system to perform expansions of arbitrary time-dependent wave functions by superposition of eigenstates of the invariant. This may be generalized to non-Hermitian Hamiltonians [38-40]. The invariants for $P T$-symmetric Hamiltonians have also been studied by Lohe [41]. We shall assume that for a Hamiltonian

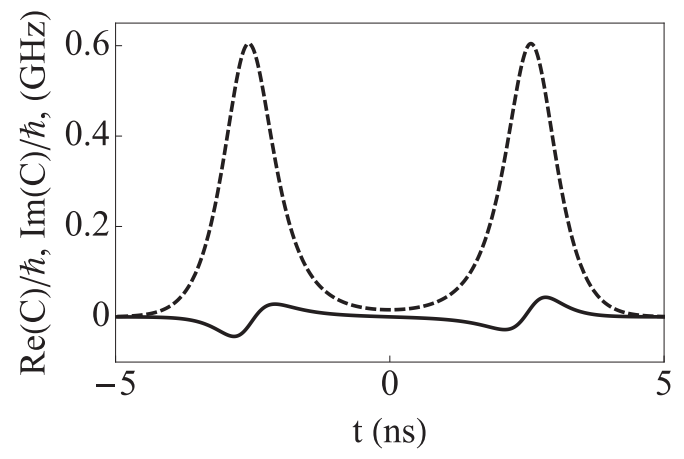

FIG. 3. Real (solid line) and imaginary (dashed line) parts of $C$. Parameters as described in the legend of Fig. 1. 
$H_{0}(t)$ with the features described in Sec. IA, there is a generalized invariant $I(t)$ that satisfies

$$
\frac{\partial I(t)}{\partial t}-\frac{i}{\hbar}\left[I(t), H_{0}(t)\right]=0
$$

so that $\frac{d}{d t}\langle\hat{\Psi}(t)|I(t)| \Psi(t)\rangle=0$. This is not an ordinary expectation value $\langle\Psi(t)|I(t)| \Psi(t)\rangle /\langle\Psi(t) \mid \Psi(t)\rangle$. In this sense, the concept of generalized invariant differs from the one for Hermitian Hamiltonians. Note also that Eq. (46) implies as well the invariance of more general matrix elements of the form $\left\langle\hat{\Psi}^{\prime}(t)|I(t)| \Psi(t)\right\rangle$, in which $\left|\hat{\Psi}^{\prime}\right\rangle$ is not necessarily the partner of $|\Psi\rangle$ but any state evolving with $H_{0}^{\dagger}$. An interesting application is put forward in the following section.

Let us assume also that $I(t)$ has a nondegenerate complete biorthonormal set of instantaneous eigenstates, $\left\{\left|\psi_{n}(t)\right\rangle,\left|\hat{\psi}_{n}(t)\right\rangle\right\}$, where $n$ varies from 1 to $N$, that satisfy

$$
\begin{gathered}
I(t)\left|\psi_{n}(t)\right\rangle=I_{n}(t)\left|\psi_{n}(t)\right\rangle, \\
I^{\dagger}(t)\left|\hat{\psi}_{n}(t)\right\rangle=I_{n}^{*}(t)\left|\hat{\psi}_{n}(t)\right\rangle, \\
\left\langle\hat{\psi}_{m}(t) \mid \psi_{n}(t)\right\rangle=\delta_{m n}, \\
\sum_{n}\left|\hat{\psi}_{n}(t)\right\rangle\left\langle\psi_{n}(t)\right|=1 .
\end{gathered}
$$

We can write the general solutions of the Schrödinger equations for $H_{0}(t)$ and $H_{0}^{\dagger}(t)$, Eqs. (8) and (9), as

$$
\begin{aligned}
& |\Psi(t)\rangle=\sum_{n} d_{n} e^{i \alpha_{n}(t)}\left|\psi_{n}(t)\right\rangle, \\
& |\hat{\Psi}(t)\rangle=\sum_{n} \hat{d}_{n} e^{i \alpha_{n}^{*}(t)}\left|\hat{\psi}_{n}(t)\right\rangle,
\end{aligned}
$$

where the coefficients $\left\{d_{n}\right\}$ and $\left\{\hat{d}_{n}\right\}$ do not depend on time, and the generalized Lewis-Riesenfeld phases are

$$
\alpha_{n}(t)=\int_{0}^{t}\left\langle\hat{\psi}_{n}\left(t^{\prime}\right)\left|i \frac{\partial}{\partial t^{\prime}}-H\left(t^{\prime}\right)\right| \psi_{n}\left(t^{\prime}\right)\right\rangle d t^{\prime} .
$$

Inverse engineering techniques rely on designing the invariant eigenvectors and phase factors first, possibly taking into account partial information on the structure of the Hamiltonian, and then deducing the Hamiltonian from them.

\section{CLASSICAL PARTICLE IN AN EXPANDING HARMONIC TRAP}

It is possible to study the motion of a classical particle with position $q(t)$ and momentum $p(t)$ in a harmonic trap as a formal quantum two-level system with non-Hermitian Hamiltonian, by rewriting the classical canonical equations of motion in matrix form [38,39]. The Hamiltonian of a classical harmonic oscillator with a time-dependent frequency $\omega(t)$ is

$$
H_{h o}(t)=\frac{p^{2}}{2 m}+\frac{1}{2} m \omega^{2}(t) q^{2}
$$

where $m$ is the mass of the particle. We shall study an expansion from $\omega_{0}=\omega(0)$ at $t=0$ to $\omega_{f}=\omega\left(t_{f}\right)$ at the final time $t=t_{f}$, with $\omega_{f}<\omega_{0}$. The corresponding classical canonical equations

$$
\begin{aligned}
& \dot{q}=\frac{\partial H_{h o}}{\partial p}=\frac{p(t)}{m}, \\
& \dot{p}=-\frac{\partial H_{h o}}{\partial q}=-m \omega^{2}(t) q(t),
\end{aligned}
$$

can be written, multiplying on both sides by $i$, as

$$
i\left(\begin{array}{c}
\dot{q} \\
\dot{p}
\end{array}\right)=i\left(\begin{array}{cc}
0 & 1 / m \\
-m \omega^{2}(t) & 0
\end{array}\right)\left(\begin{array}{c}
q(t) \\
p(t)
\end{array}\right),
$$

due to their linear dependence on $q$ and $p$. This has the form of a Schrödinger-like equation $(\hbar=1)$ with the "effective" non-Hermitian Hamiltonian

$$
\mathcal{H}(t)=i\left(\begin{array}{cc}
0 & 1 / m \\
-m \omega^{2}(t) & 0
\end{array}\right)
$$

and "state vector"

$$
|\psi\rangle=\left(\begin{array}{c}
q(t) \\
p(t)
\end{array}\right) .
$$

The analogy is useful, as we shall see, but formal, since this Hamiltonian does not have units of energy, in fact different matrix elements have different units as the "state vector" components $q$ and $p$ have also different units. Moreover, the usual interpretational rules for the formalism of a quantum mechanical two-level system do not apply here. For example, the state-vector components contain the information of a classical trajectory and have nothing to do with probability amplitudes. Also, unlike the quantum state, which requires normalization, the classical state can be multiplied by any constant, and each constant implies a different classical trajectory and a different total energy. In spite of these strong interpretational discrepancies, the analogy has a practical value, as it allows us to express the classical dynamics "a la quantum," making use of linear superposition of eigenstates and matrix operations. We may thus apply the generalized invariant theory and expand the "state vector" in terms of formal eigenvectors of the generalized invariants to apply inverse engineering methods and design a trap expansion without final excitation. Defining the generalized invariant matrix as [39]

$$
\mathcal{I}(t)=\left(\begin{array}{cc}
b(t) & c(t) \\
-a(t) & -b(t)
\end{array}\right),
$$

and imposing Eq. (46), without $\hbar$, we find

$$
\begin{aligned}
& a(t)=m\left[\frac{\omega_{0}}{\varrho^{2}(t)}+\frac{1}{\omega_{0}} \dot{\varrho}^{2}(t)\right], \\
& b(t)=\frac{-1}{\omega_{0}} \varrho(t) \dot{\varrho}(t), \\
& c(t)=\frac{\varrho^{2}(t)}{\omega_{0} m},
\end{aligned}
$$

where the dimensionless scaling function $\varrho(t)$ satisfies the auxiliary equation

$$
\ddot{\varrho}(t)+\omega^{2}(t) \varrho(t)=\frac{\omega_{0}^{2}}{\varrho^{3}(t)} .
$$


This is the Ermakov equation, the same equation for the scaling function that defines the invariants in the expansion of the quantum harmonic oscillator [13].

The physical meaning of the generalized invariant matrix is better appreciated by noticing that matrix elements of the form $\left\langle\hat{\psi}^{\prime}(t)|\mathcal{I}(t)| \psi(t)\right\rangle$, where $\left|\hat{\psi}^{\prime}\right\rangle$ is any vector evolving with $\mathcal{H}^{\dagger}$, remain constant. Since Hamilton's equations, Eq. (57), may also be written as

$$
i\left(\begin{array}{c}
-\dot{p} \\
\dot{q}
\end{array}\right)=\mathcal{H}^{\dagger}\left(\begin{array}{c}
-p(t) \\
q(t)
\end{array}\right),
$$

we notice that the classical trajectory written as

$$
\left|\hat{\psi}^{\prime}(t)\right\rangle=\left(\begin{array}{c}
-p(t) \\
q(t)
\end{array}\right)
$$

evolves according to the Hermitian adjoint Hamiltonian $\mathcal{H}^{\dagger}$. The matrix element $\left\langle\hat{\psi}^{\prime}(t)|\mathcal{I}(t)| \psi(t)\right\rangle$ with Eqs. (59) and (66) gives then the known quadratic Ermakov invariant for the time-dependent harmonic oscillator defined up to an arbitrary constant by a combination of terms proportional to $p^{2}, q^{2}$, and $q p$; see, e.g., Refs. [13,42].

For $\mathcal{I}(t)$, whose eigenvalues are $I_{ \pm}= \pm i$, the eigenstates are

$$
\left|\psi_{ \pm}(t)\right\rangle=\left(\begin{array}{c}
c(t) \\
\pm i[1 \pm i b(t)]
\end{array}\right)
$$

These eigenstates may be regarded as intermediary elements of the calculation, but, unlike other applications of the invariant theory to true quantum systems, they do not have, when isolated, a direct physical trajectory realization, which requires real position and momentum.

Setting also the partners

$$
\left|\hat{\psi}_{ \pm}(t)\right\rangle=\left(\begin{array}{c}
\frac{a(t) / 2}{1 \mp i b(t)} \\
\pm \frac{i}{2}
\end{array}\right)
$$

the Lewis-Riesenfeld phases $\alpha_{ \pm}(t)$, Eq. (53), are now

$$
\begin{aligned}
\alpha_{ \pm}(t) & =\int_{0}^{t}\left\langle\hat{\psi}_{ \pm}\left(t^{\prime}\right)\left|i \frac{\partial}{\partial t^{\prime}}-\mathcal{H}\left(t^{\prime}\right)\right| \psi_{ \pm}\left(t^{\prime}\right)\right\rangle d t^{\prime} \\
& =i \ln \sqrt{\frac{c(t)}{c(0)}} \pm \omega_{0} \int_{0}^{t} \frac{1}{\varrho^{2}\left(t^{\prime}\right)} d t^{\prime} .
\end{aligned}
$$

Then, the phase-space trajectory is given by solving the formal Schrödinger equation, making use of the superposition of eigenvectors of the generalized invariant,

$$
\begin{aligned}
\left(\begin{array}{c}
q(t) \\
p(t)
\end{array}\right) & =d_{+} e^{i \alpha_{+}(t)}\left|\psi_{+}(t)\right\rangle+d_{-} e^{i \alpha_{-}(t)}\left|\psi_{-}(t)\right\rangle \\
& =R\left(\begin{array}{c}
\varrho(t) \cos \theta(t) \\
-\frac{m \omega_{0}}{\varrho(t)} \sin \theta(t)+m \varrho(t) \cos \theta(t)
\end{array}\right),
\end{aligned}
$$

where $R=2 r \sqrt{c(0) / m \omega_{0}}$ is the position amplitude of the trajectory for the trap fixed at the initial frequency, $d_{+}=d_{-}^{*}=$ $r \exp \left(i \theta_{0}\right)$ can be determined by the initial conditions at $t=0$, and

$$
\theta(t)=\omega_{0} \int_{0}^{t} \frac{1}{\varrho^{2}\left(t^{\prime}\right)} d t^{\prime}+\theta_{0},
$$

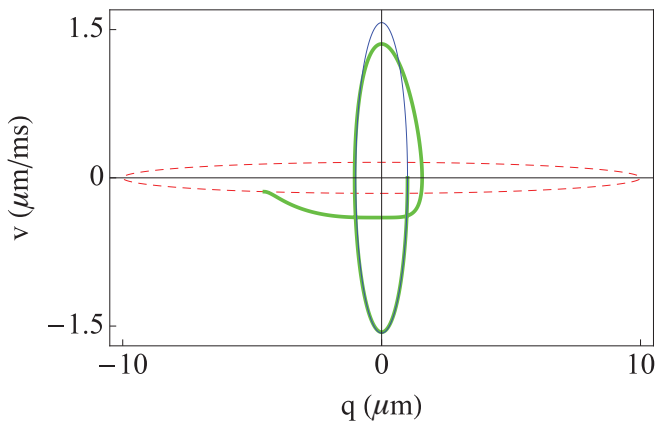

FIG. 4. (Color online) Parametric velocity-position trajectory. The initial ellipse (solid blue thin line) and the final ellipse (red dashed line) are connected by the shortcut trajectory (green solid thick line). Parameters: $\theta_{0}=0$ corresponding to $q_{0}=q(t=0)=1 \mu \mathrm{m}$ and $v_{0}=$ $v(t=0)=0 \mu \mathrm{m} / \mathrm{ms}, \omega_{0}=2 \pi \times 250 \mathrm{~Hz}, \omega_{f}=2 \pi \times 2.5 \mathrm{~Hz}, t_{f}=$ $25 \mathrm{~ms}$, and the mass of an atom of rubidium- $87, m=1.44 \times 10^{-25} \mathrm{~kg}$.

with $\theta_{0}$ the initial phase. ${ }^{1}$ Equation (70) is the important result that relates the classical trajectory to the scaling function $\varrho(t)$. In the inverse method, we can manipulate and design $\varrho(t)$ so as to achieve a desired objective. $\omega(t)$ will be then obtained from the Ermakov equation, Eq. (64).

Imposing the boundary conditions $\varrho(0)=1$ and $\dot{\varrho}(0)=0$, and $\varrho\left(t_{f}\right)=\left(\omega_{0} / \omega_{f}\right)^{1 / 2}$ and $\dot{\varrho}\left(t_{f}\right)=0$, which consistently with the Ermakov equation imply $\ddot{\varrho}(0)=\ddot{\varrho}\left(t_{f}\right)=0$, we find for the energy $E_{0}:=E(t=0)=\omega_{0}^{2} R^{2} m / 2$ and $E_{f}:=$ $E\left(t_{f}\right)=\omega_{f} E_{0} / \omega_{0}$. In other words, these boundary conditions guarantee that the value of the classical adiabatic invariant $E(t) / \omega(t)$ at initial and final times coincides, even though it may take different values at intermediate times. This is the meaning of "no final excitation" in this context.

To design the expansion process fully, $\varrho(t)$ has to be interpolated at intermediate times. We use here for simplicity a polynomial form, $\varrho(t)=\sum_{n=0}^{5} r_{n} t^{n}$, where the coefficients $r_{n}$ are fixed from the boundary conditions. Then we get $\omega(t)$ from the Ermakov equation,

$$
\omega(t)=\sqrt{\frac{\omega_{0}^{2}}{\varrho^{4}(t)}-\frac{\ddot{\varrho}(t)}{\varrho(t)}} .
$$

In Fig. 4, we have represented a shortcut trajectory in phase space between the initial and final times, $t=0$ and $t=t_{f}$, for the frequency $\omega(t)$ given by Eq. (72). We have also added to the trajectory a period $T_{0}=2 \pi / \omega_{0}$ before $t=0$, and a period $T_{f}=2 \pi / \omega_{f}$ after $t=t_{f}$, for which the particle evolves for fixed $\omega_{0}$ and $\omega_{f}$, respectively, so as to depict complete initial and final phase-space ellipses. It is clear from the figure that the shortcut trajectory that connects the initial and final ellipses is not an adiabatic path. Such a path would be formed by a succession of ellipses slowly varying from the initial to the final ones.

\footnotetext{
${ }^{1}$ The linear invariants of the classical harmonic oscillator $[41,42]$ could be obtained from $\left\langle\hat{\Psi}^{\prime}|\mathcal{I}| \Psi\right\rangle$ by using instead of Eqs. (59) or (66) one of the eigenvectors of $\mathcal{I}$ times the corresponding LewisRiesenfeld phase factor. This takes us away from our current objective so we shall not discuss it further.
} 


\section{DISCUSSION AND CONCLUSION}

We have generalized shortcut to adiabaticity techniques for non-Hermitian Hamiltonian systems and provided application examples. Experimental implementations are at reach both for internal state population inversion and time-dependent harmonic oscillators. Related open questions are the application of similar concepts to master equations, or developing means to implement arbitrary non-Hermitian interactions. Another interesting research avenue is to combine shortcut techniques with optimal control [19,23] taking into account physically imposed constraints.

The present work bears some common elements with the quantum brachistochrone problem [43] solved with nonHermitian Hamiltonians [44-48], so it is worth stressing the similarities and differences. In the brachistochrone problem, an initial state $\left|\psi_{I}\right\rangle$ and a final state $\left|\psi_{F}\right\rangle$ are fixed, and the objective is to find the Hamiltonian (typically time independent) that carries $\psi_{I}$ to $\psi_{F}$ in the shortest possible time subject to a constrained variance. If the Hamiltonian is Hermitian, this leads to the minimal time given by Fleming's bound [49], whereas if non-Hermitian Hamiltonians are allowed, the process time can go to zero. This has been worked out in detail in particular for the so-called $P T$-symmetric Hamiltonians with real eigenvalues $[44,45,47,48]$, although the phenomenon is more general [46]. The problem that remains is to physically realize the resulting Hamiltonian, and a way out could be to embed the system into a larger one by using the Naimark dilation theorem [50].

Among the differences with the present work let us point out first some that apply independently of the use of nonHermitian Hamiltonians: in the brachistochrone problem, the initial and final states are fixed so in general the optimized Hamiltonian depends on them, and no reference is made to an adiabatic process. In a shortcuts-to-adiabaticity approach, the starting point is instead a given adiabatic process defined by a
Hamiltonian with time-dependent parameters. Typically, the boundary conditions at initial and final times are thus set by an initial and a final form of the Hamiltonian, and the goal is to carry out any initial state from initial to final configurations without final excitation, faster than the reference adiabatic process. The constraints that may be applied to choose among or optimize the shortcut paths may vary depending on the physical system and are not limited to the energy variance. The energy itself may need to be bounded, to avoid an excessive transient excitation. In a transport problem, for example, we might also want or need to impose constraints on the trajectory domain in physical space [26,27]. Obviously, several constraints may also be applied to the quantum brachistochrone [43].

As for the nonhermiticity of the Hamiltonian, in the brachistochrone it is proposed as a way to provide dynamics faster than Hermitian. Here, we start instead with a physical system that is effectively described by a non-Hermitian Hamiltonian and look for a new Hamiltonian, using different inverse techniques, that allows us to reproduce more quickly the results of a slow adiabatic process.

As an example of the useful relations between the brachistochrone and the present work, note that the difficulty to realize a given NH-Hamiltonian, a common potential problem of the brachistochrone and the shortcuts, may be solved by similar means, e.g., by enlarging the system with the aid of Naimark extensions [50].

\section{ACKNOWLEDGMENTS}

We acknowledge funding by the Basque Government (Grant No. IT472-10) and Ministerio de Ciencia e Innovación (FIS2009-12773-C02-01). E.T. and S.I. acknowledge financial support from the Basque Government (Grants No. BFI08.151 and BFI09.39). X.C. acknowledges support from Juan de la Cierva Programme and the National Natural Science Foundation of China (Grant No. 60806041).
[1] M. Demirplak and S. A. Rice, J. Phys. Chem. A 107, 9937 (2003).

[2] M. Demirplak and S. A. Rice, J. Phys. Chem. B 109, 6838 (2005).

[3] M. Demirplak and S. A. Rice, J. Chem. Phys. 129, 15411 (2008).

[4] R. Reichle, D. Leibfried, R. B. Blakestad, J. Britton, J. D. Jost, E. Knill, C. Langer, R. Ozeri, S. Seidelin, and D. J. Wineland, Fortschr. Phys. 54, 666 (2006).

[5] A. Couvert, T. Kawalec, G. Reinaudi, and D. Guéry-Odelin, Europhys. Lett. 83, 13001 (2008).

[6] S. Masuda and K. Nakamura, Phys. Rev. A 78, 062108 (2008).

[7] J. G. Muga, X. Chen, A. Ruschhaupt, and D. Guéry-Odelin, J. Phys. B 42, 241001 (2009).

[8] J. Grond, J. Schmiedmayer, and U. Hohenester, Phys. Rev. A 79, 021603 (2009).

[9] J. Grond, G. von Winckel, J. Schmiedmayer, and U. Hohenester, Phys. Rev. A 80, 053625 (2009).

[10] M. V. Berry, J. Phys. A 42, 365303 (2009).
[11] P. Salamon, K. H. Hoffmann, Y. Rezek, and R. Kosloff, Phys. Chem. Chem. Phys. 11, 1027 (2009).

[12] M. Murphy, L. Jiang, N. Khaneja, and T. Calarco, Phys. Rev. A 79, 020301(R) (2009).

[13] X. Chen, A. Ruschhaupt, S. Schmidt, A. del Campo, D. Guéry-Odelin, and J. G. Muga, Phys. Rev. Lett. 104, 063002 (2010).

[14] X. Chen, I. Lizuain, A. Ruschhaupt, D. Guéry-Odelin, and J. G. Muga, Phys. Rev. Lett. 105, 123003 (2010).

[15] X. Chen and J. G. Muga, Phys. Rev. A 82, 053403 (2010).

[16] J. F. Schaff, X. L. Song, P. Vignolo, and G. Labeyrie, Phys. Rev. A 82, 033430 (2010); 83, 059911(E) (2011).

[17] S. Masuda and K. Nakamura, Proc. R. Soc. A 466, 1135 (2010).

[18] J. G. Muga, X. Chen, S. Ibáñez, I. Lizuain, and A. Ruschhaupt, J. Phys. B 43, 085509 (2010).

[19] D. Stefanatos, J. Ruths, and Jr-Shin Li, Phys. Rev. A 82, 063422 (2010)

[20] J. F. Schaff, X. L. Song, P. Capuzzi, P. Vignolo, and G. Labeyrie, Europhys. Lett. 93, 23001 (2011). 
[21] J. F. Schaff, P. Capuzzi, G. Labeyrie, and P. Vignolo, e-print arXiv:1105.2119v1.

[22] X. Chen, E. Torrontegui, and J. G. Muga, Phys. Rev. A 83, 062116 (2011)

[23] B. Andresen, K. H. Hoffmann, J. Nulton, A. Tsirlin, and P. Salamon, Eur. J. Phys. 32, 827 (2011).

[24] Y. Li, L.-A. Wu, and Z.-D. Wang, Phys. Rev. A 83, 043804 (2011).

[25] A. del Campo, e-print arXiv:1103.0714.

[26] E. Torrontegui, S. Ibáñez, X. Chen, A. Ruschhaupt, D. GuéryOdelin, and J. G. Muga, Phys. Rev. A 83, 013415 (2011).

[27] E. Torrontegui, X. Chen, M. Modugno, S. Schmidt, A. Ruschhaupt, and J. G. Muga, e-print arXiv:1103.2532.

[28] J. G. Muga, J. P. Palao, B. Navarro, and I. L. Egusquiza, Phys. Rep. 395, 357 (2004).

[29] J. C. Garrison and E. M. Wright, Phys. Lett. A 128, 177 (1988).

[30] A. Mostafazadeh, J. Math. Phys. 40, 3311 (1999).

[31] M. V. Berry, Proc. R. Soc. London A 392, 45 (1984).

[32] L. I. Schiff, Quantum Mechanics (McGraw-Hill, New York, 1981).

[33] E. W. Streed, J. Mun, M. Boyd, G. K. Campbell, P. Medley, W. Ketterle, and D. E. Pritchard, Phys. Rev. Lett. 97, 260402 (2006).

[34] J. G. Muga, J. Echanobe, A. del Campo, and I. Lizuain, J. Phys. B 41, 175501 (2008).
[35] S. Ibáñez, A. Peralta Conde, D. Guéry-Odelin, and J. G. Muga, Phys. Rev. A 84, 013428 (2011).

[36] F. Bloch, Phys. Rev. 70, 460 (1946).

[37] H. R. Lewis and W. B. Riesenfeld, J. Math. Phys. 10, 1458 (1969).

[38] X. C. Gao, J. B. Xu, and T. Z. Qian, Phys. Rev. A 44, 7016 (1991).

[39] X. C. Gao, J. B. Xu, and T. Z. Qian, Phys. Rev. A 46, 3626 (1992).

[40] T. Z. Qian, X. C. Gao, and J. B. Xu, Phys. Rev. A 48, 11401 (1993).

[41] M. A. Lohe, J. Phys. A: Math. Theor. 42, 035307 (2009).

[42] H. R. Lewis and P. G. Leach, J. Math. Phys. 23, 2371 (1982).

[43] A. Carlini, A. Hosoya, T. Koike, and Y. Okudaira, Phys. Rev. Lett. 96, 060503 (2006).

[44] C. M. Bender, D. C. Brody, H. F. Jones, and B. K. Meister, Phys. Rev. Lett. 98, 040403 (2007).

[45] A. Mostafazadeh, Phys. Rev. Lett. 99, 130502 (2007).

[46] P. E. G. Assis and A. Fring, J. Phys. A: Math. Theor. 41, 244002 (2008).

[47] A. Mostafazadeh, Phys. Rev. A 79, 014101 (2009).

[48] C. M. Bender and D. C. Brody, Lect. Notes Phys. 789, 341 (2009).

[49] G. N. Fleming, Nuovo Cimento Soc. Ital. Fis. A 16, 232 (1973).

[50] U. Günther and B. F. Samsonov, Phys. Rev. Lett. 101, 230404 (2008). 